\title{
Study on thermal insulation of diagonal- braced wood frame wall
}

Mingbin Liu, Feng Lu, Xiaolin Yang, Yang ruyuan

This preprint has been removed because it was erroneously supplied by a journal. 